\title{
Protrusions in Graphs and Their Applications
}

\author{
Fedor V. Fomin \\ Department of Informatics, Univeristy of Bergen, 5020 Bergen, Norway \\ fomin@ii.uib.no
}

A protrusion in a graph is a subgraph of constant treewidth that can be separated from the graph by removing a constant number of vertices. More precisely, given a graph $G=(V, E)$ and $S \subseteq V$, we denote by $\partial(S)$ the set of vertices in $S$ that have a neighbour in $V \backslash S$.

Definition 1. Given a graph $G=(V, E)$, we say that a set $X \subseteq V$ is an $r$ protrusion of $G$ if $|\partial(X)| \leq r$ and the treewidth of the subgraph $G[X]$ of $G$ induced by $X$ is at most $r$.

Protrusions were introduced in [1] as a tool for obtaining kernelization metatheorems. Loosely speaking, if a parametrized problem can be expressed in a certain logic, then a sufficiently large protrusion can be replaced by a smaller "equivalent" one. Protrusions appear to be a handy tool for obtaining kernelization and approximation algorithms [2]. In this talk we discuss combinatorial properties of graphs implying existence of large protrusions and give a number of algorithmic applications of protrusions.

The talk is based on joint works with Hans L. Bodlaender, Daniel Lokshtanov, Elko Penninkx, Saket Saurabh, Venkatesh Raman, and Dimitrios M. Thilikos 123 .

\section{References}

1. Bodlaender, H., Fomin, F.V., Lokshtanov, D., Penninkx, E., Saurabh, S., Thilikos, D.M.: (Meta) Kernelization. In: Proceedings of the 50th Annual Symposium on Foundations of Computer Science (FOCS 2009), pp. 629-638. IEEE, Los Alamitos (2009)

2. Fomin, F.V., Lokshtanov, D., Saurabh, S., Thilikos, D.M.: Bidimensionality and kernels. In: Proceedings of the 21th ACM-SIAM Symposium on Discrete Algorithms (SODA 2010), pp. 503-510 (2010)

3. Fomin, F.V., Lokshtanov, D., Raman, V., Saurabh, S.: Bidimensionality and EPTAS, CoRR abs/1005.5449 (2010) 